AUTHOR(S):

SUBMITTED TO:
ON PARALLEL SUPERCOMPUTERS

Michael S. Warren, T-6

Wojciech H. Zurek, T-6

Peter J. Quinn, Mt. Stromlo Obs., Australia

J. K. Salmon, Caltech

Lef $\$ 39990$ 


\title{
THE SHAPE OF THE INVISIBLE HALO: $N$-BODY SIMULATIONS ON PARALLEL SUPERCOMPUTERS
}

\author{
M. S. Warren, W. H. Zurek \\ Theoretical Astrophysics, T-6, Mail Stop B288 \\ Los Alamos National Laboratory' \\ Los Alamos, New Mexico 87545 \\ P. J. Quinn \\ Mount Stromlo and Siding Springs Observatories \\ The Australian National University \\ P.B. Weston Creek, A.C.T. 2611, Australia \\ J. K. Salmon \\ Caltech Concurrent Supercomputing Facility \\ California Institute of Technology, 206-49 \\ Pasadena, California 91126
}

\begin{abstract}
ABSTR.ACT
We study the shapes of halos and the relationship to their angular momentum content by means of $\mathrm{N}$-body $\left(\mathrm{N} \sim 10^{6}\right)$ simulations. Results indicate that in relaxed halos with no apparent substructure:

i.) The shape and orientation of the isodensity contours tends to persist throughout the virialised portion of the halo.

ii.) Most $(\approx 70 \%)$ of the halos are prolate.

iii.) The approximate direction of the angular momentum vector tends to persist throughout the halo.

iv.) For spherical shells centered on the core of the haio the magnitude of the specific angular momentum is approximately proportional to their radius.

v.) The shortest axis of the ellipsoid which approximates the shape of the halo tends to align with the rotation axis of the halo. This tendency is strongest in the fastest rotating halos.
\end{abstract}

\section{INTRODUCTION}

We model the formation of collapsed structures in an Einstein-deSitter $(\Omega=1)$ universe. The initial power spectrum of density perturbations was:

$$
P(k)= \begin{cases}A k_{b}^{-3}\left(\frac{k}{k_{b}}\right)^{-2}, & \text { if } k>k_{b} \\ A k_{b}^{-3}\left(\frac{k}{k_{b}}\right), & \text { if } k<k_{b} .\end{cases}
$$

The wavelength at which the crossover occurs is $2 \pi / k_{b} \approx 1.5 \mathrm{Mpc}$. This spectrum was chosen to investigate the inheritance of features on sub-galactic scales. The initial conditions were produced by Fourier transforming the initial complex amplitudes (chosen from a Gaussian random distribution) and using the result to perturb a $128^{3}$ mesh of particles according to the Zel'dovich growing mode formalism..$^{1,2}$ The computational volume was a sphere of comoving radius $5 \mathrm{Mpc}$ cut out from this cube. The initial conditions correspond to a redshift $z=15$, and the simulation was terminated after expansion by a factor of 20 . Vacuum boundary conditions were used, with a buffer region of $1.5 \mathrm{Mpc}$ at the outer edge which was excluded from analysis. The power spectrum was normalized such that $\delta M / M$ was initially 0.1 at a scale of $1 \mathrm{Mpc}$. The equations of motion were solved 
in physical coordinates, with a time variable $p=t^{0.75}$. The Newtonian force law was smoothed at small scales by a Plummer kernel with a softening length (in physical coordinates) of $5 \mathrm{kpc}$. The total mass of the system was $3.5 \times 10^{13} M_{\odot}$ and 1097921 particles were used. All results are presented in units which assume a Hubble constant of $50 \mathrm{~km} \mathrm{sec}^{-1} \mathrm{Mpc}^{-1}$.

Two aspects of the simulation depend greatly on the number of particles. The initial conditions are at best accurate down to a scale of the mean interparticle spacing. Aiso, analysis of other parameters of the halos is affected by $1 / N^{3 / 2}$ noise. Because the final halos in this simulation contain $\sim 10^{4}$ particles, their parameters may be determined fairly reliably.

\section{THE PARALLEL TREECODE}

A modified version of the Barnes-Hut treecode ${ }^{3}$ was used to simulate the dissipationless collapse of objects from the initial conditions. The basic method creates an oct-tree subdivision of space to group particles. The force is calculated by descending the hierarchical tree. This results in an execution time which scales with the number of particles as $N \log N$. The performance and accuracy of hierarchical tree methods in the cosmological context has been investigated by Bouchet and Hernquist. ${ }^{4}$ In the original Barnes-Hut algorithm the size of the cell, $s$, is compared to the distance from the particle to the center of mass of the cell, $d$. If $s / d \leq \theta$ where $\theta$ is an adjustable tolerance parameter, the particles within the cell are treated as a single psuedo-particle. The results presented here use a treecode which differs in its definition of $\theta$. The new parameter, $\theta_{f d}$, uses the distance from the particle to the nearest point on the surface of the cell (face-distance) in place of $d$. This corrects a problern in the Barnes-Hut algorithm which occurs when a dense object enters the corner of a cell which has a significant mass concentration in the opposite corner. ${ }^{5}$ We have used $\theta_{\rho d}=1.6$, (roughly equivalent to a BarnesHut $\theta=0.75)$ with quadrupole cell interactions.

Our parallel code runs on several message passing, distributed memory parallel computers (hypercubes). This type of machine typically has between 32 and 1024 processors, each with speed and memory comparable to a typical workstation. Computations presented here were done on the 64 processor Caltech/JPL MarkIII hypercube. Each node of the machine contains two Motorola 68000 series microprccessors, along with a 64 bit Weitek floating point unit capable of nearly 3 million single precision floating point calculations per second. The lowest level of the force calculation routine was coded in Weitek assembly language to achieve this performance. Each processing unit has 4 million bytes of local memory, giving a total of 256 Mbytes of memory. The 900 timesteps in the simulation, consisting of $35 \times 10^{12}$ floating point operations, were completed in about 60 hours of CPU time. The time needed per timestep for the calculation ranged from 190 sec. at $z=15$, to $280 \mathrm{sec}$. at $z=-0.3$, demonstrating that the computing time is relatively insensitive to the amount of clustering in the system, in contrast to particle-particle particle-mesh methods.

The efficiency of a parallel program may be established by comparing it to an extrapolated version of a sequential computation. This quantifies the amount of processing speed lost to parallel overhead. The parallel overhead may be further broken down into load imbalance and communication overhead. The time spent sending messages between processors in the code is negligible, and the overall efficiency is close to $85 \%$ with 64 processors. This means the program runs only $15 \%$ slower than it would on a hypothetical sequential computer with computing power $P_{64}=64 \times P_{1}$, where $P_{1}$ is the power of a single processor node. It is fortunate that the nature of the treecode algorithm, which is inefficient on vector 
supercomputers, fits very well within the scheme of "large-grained" parallelism.

In order to allow each processor to take part in the overall computation, a technique termed domain decomposition is used. Space is divided up into distinct subvolurnes, with each processor responsible for updating the positions of each particle in its region. The straightforward approach of dividing space into a cubical mesh is not feasible, as some regions of space will have many more particles, making some processors do all the work, while others sit idle. The method actually used divides space into irregular cells which gives each processor an equal amount of work, so that the load imbalance inefficiency is minimized. ${ }^{5}$

The parallel treecode is still being developed, and there are many improvements yet to be made. The most pressing is the integration of smoothed particle hydrodynamics into the equations of motion to study how dissipation affects the formation of structure. Additional modification of the subdivision criterion will certainly improve accuracy, and a better integrator would also be welcome. The addition of two-body regularization and a locally variable timestep would also allow applications to globular clusters. There is also the inevitable improvement in parallel supercomputers. Parallel machines are now abreast of the vector supercomputers, and their performance is improving more rapidly.

The practice of quoting energy conservation as a test of accuracy in a cosmological simulation is ambiguous. For $\Omega=1$ the total energy is very near zero, so comparing the error to this value is of little use. If the error is compared to the potential energy, it must be specified at a given time, relative to either the physical potential energy, or the cosmic potential energy. For this simulation, if the energy is defined in physical coordinates, the ratio of the error at the end of the simulation, divided by the potential energy at the end, was slightly less than $1 \%$.

\section{CHARACTERIS'TICS OF HALOS}

Halos were chosen with a two part algorithm. First all regions in a 3D mesh with an overdensity of 2000 or more were identified. Then these regions were collected into groups that were separated by at least $300 \mathrm{kpc}$ from a neighboring group. Particles within $300 \mathrm{kpc}$ of the center of mass of the group were then collected as a single halo. The exact center of the halo was determined by the mean position of the 10 most tightly bound particles. Visual inspection of the 155 halos selected in this manner identified a few objects where substructure confused the algorithm, and these were eliminated from further analysis.

Using the methods above provided 140 halos to study, ranging in mass from slightly less than $2 \times 10^{10} M_{\odot}$ to $34 \times 10^{10} M_{\odot}$. A part of the simulation volume with about 20 halos is shown in Fig. 1a. The halo indicated by the small box is examined in further detail in Figs. 2 and 4 . The volume represented in the upper left panel of Fig. 2 is $7 \times 10^{5}$ times smaller than that in Fig. la, yet it still contains almost 2000 particles.

\section{(a.) Density profiles and rotation curves}

Previous numerical results have indicated that the mass profiles of galactic halos are related to the power law exponent of the initial density fluctuations, and that the dark halos of galaxies carry a strong memory of their initial conditions. ${ }^{9,10}$ One goal of this simulation was to look for these effects when using different power laws on small and large scales. The mass profiles of the halos may be established by plotting a rotation curve, defined by $v(r)=\sqrt{G M(r) / r}$, where $M(r)$ is the mass within radius $r$ of the center of the halo. These are shown in Fig. $1 b$. It is 

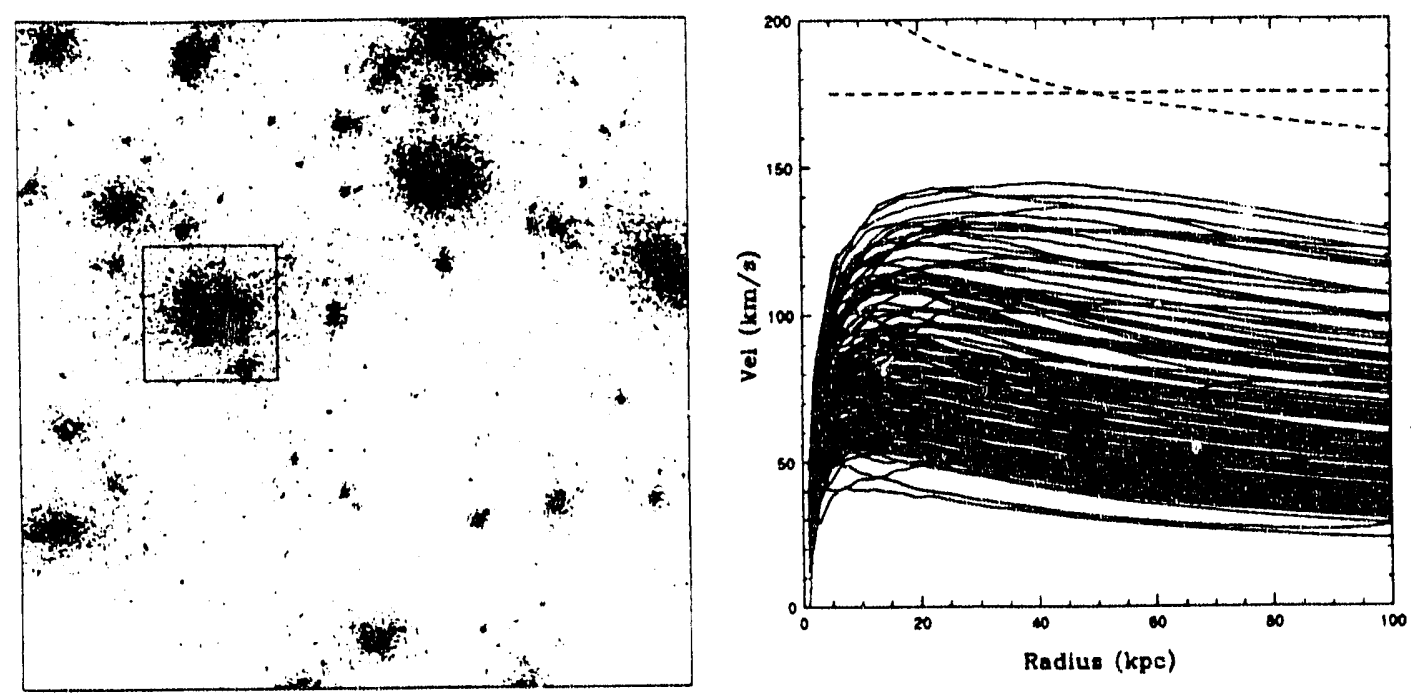

Figure 1. a.) A projected subsection volume $3 \times 3 \mathrm{Mpc}$ in area and 10 Mpc deep, which is $\approx 1 / 10$ of the entire volume. Only one of every two particles are plotted. The small box indicates a halo, the time evolution of which is illustrated in Fig. 4. Its structure is analysed in Fig. 2.

b.) Rotation curves for the 140 most massive halos. The slope predicted by the secondary infall paradigm ${ }^{6,7,8}$ for a pure Zel'dovich $(P(k) \sim k)$ power law is shown by the downwardly sloping dotted line (normalized to $1.00 \mathrm{~km} \mathrm{sec}^{-1}$ at $50 \mathrm{kpc}$, and offset vertically by $\left.75 \mathrm{~km} \mathrm{sec}^{-1}\right)$. The flat slope expected from $P(k) \sim k^{-2}$ is also indicated. ${ }^{7,8,9}$

immediately evident that the behavior of all the halos follows the same basic pattern. The slope predicted by the secondary infall paradigm ${ }^{6,7,8}$ for a pure $P(k) \sim k$ power law is shown by the downwardly sloping dotted line in Fig. $1 b$. The slope of every relaxed halo is consistent with this slope at radii larger than around $50 \mathrm{kpc}$. Each halo formed from a region which was, on average, $1 \mathrm{Mpc}$ across. The power spectrum for scales smaller than $1.5 \mathrm{Mpc}$ was $P(k) \sim k^{-2}$, so it is interesting that the behavior of the slope for a large part of the halo seems to ignore the power on scales smaller than the initial extent of the particles in the halo. There also appears to be a trend for more massive halos to have a larger core, with a more extended flat region. This effect may be related to the merger history of the halo. It is hard to tell if there is a signature of the broken power law in the rotation curves. Further simulations should provide the comparisons necessary to resolve any systematic effects. It is not even completely clear if the soft cores, where the rotation curves are rising, are a real physical result, or the result of the softened potential or relaxation effects.

\section{(b.) The shape of the halo}

In order to determine the shapes and orientations of halos, a convergent method was used to find an ellipsoidal isodensity contour constrained to have a given enclosed volume, $V=(4 \pi / 3) a b c$ where $a, b, c$ are the minor, intermediate, and major axes of the ellipsoid, respectively. The method is equivalent to finding 

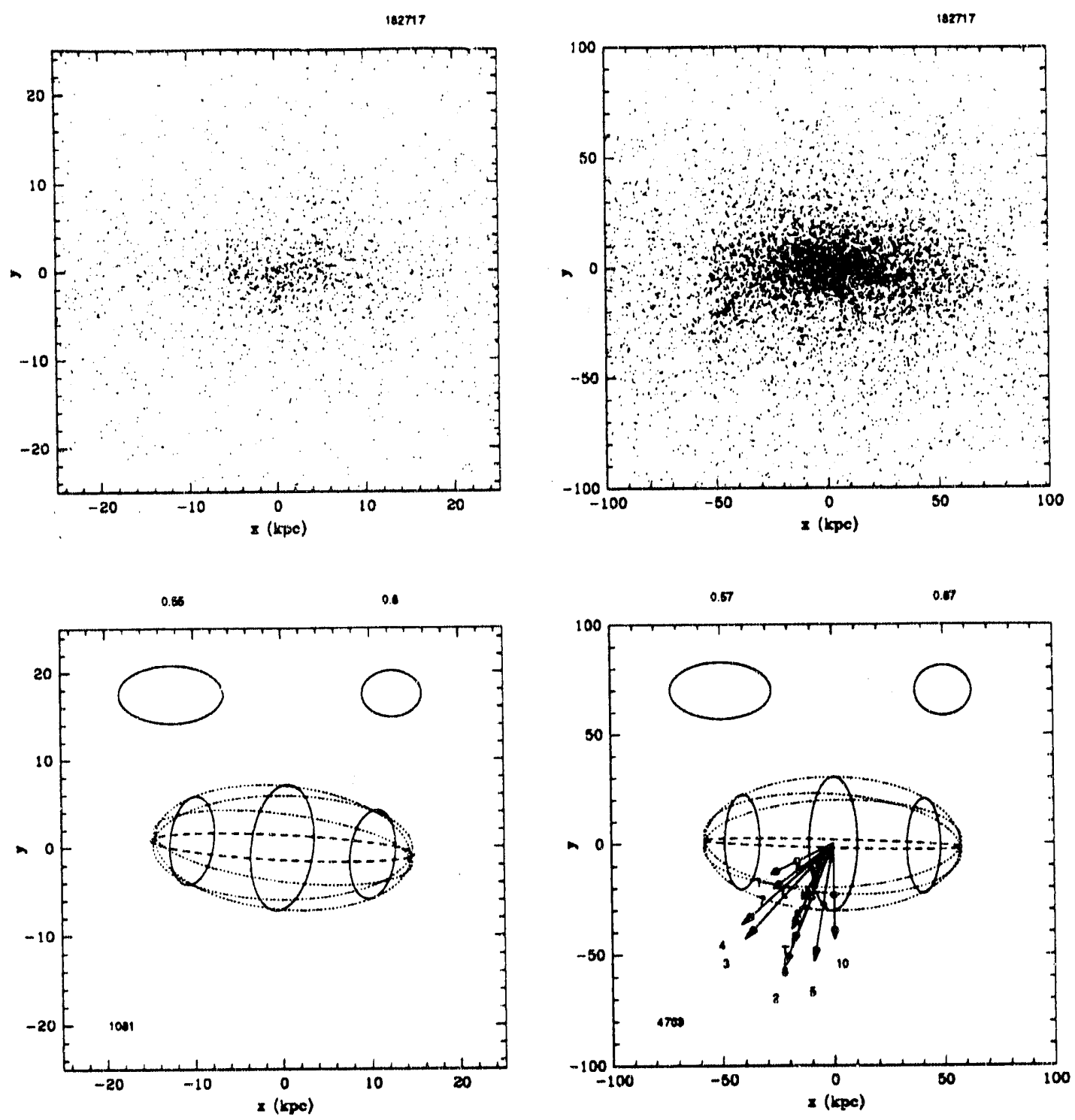

Figure 2. The top two panels show particles in a selected halo at two resolutions, a cube $50 \mathrm{kpc}$ on a side, and a cube $200 \mathrm{kpc}$ on a side. The lower panels show ellipsoids which are a best fit to an isodensity contour of the particle distribution. The ellipses indicate the projected view from along the minor and major axis of the ellipsoid. The arrows in the lower right panel indicate the direction of the angular momentum vector for particles in ten concentric spherical shells, with thickness $10 \mathrm{kpc}$. Note that the ellipsoids have the same general shape and orientation, and that the angular momentum vectors tend to point along the minor axis of the ellipsoid.

an ellipsoid which maximizes the number of particles contained within this fixed volume, centered about the previously defined center of the halo. The ellipsoids studied had volumes of spheres with radii of 10,20 , and $40 \mathrm{kpc}$. Plots were made for each halo to assess the alignment of the isodensity contours. In a large majority of the cases these contours maintain their basic shape and alignment. One 
representative example is shown in Fig. 2. The contours at 10 and $40 \mathrm{kpc}$ are shown, along with the particle distribution at two resolutions.

If the ratios of the axes are plotted against each other (see Fig. $3 a$ ) about $2 / 3$ of the halos lie above the line $e_{1}=e_{2}\left(e_{1}=b / c, e_{2}=a / b\right)$. Purely prolate objects have $e_{2}=1$, and purely oblate have $e_{1}=1$. This result may be affected by halos which have not completely relaxed, as an object with two density centers would be identified as a prolate halo.
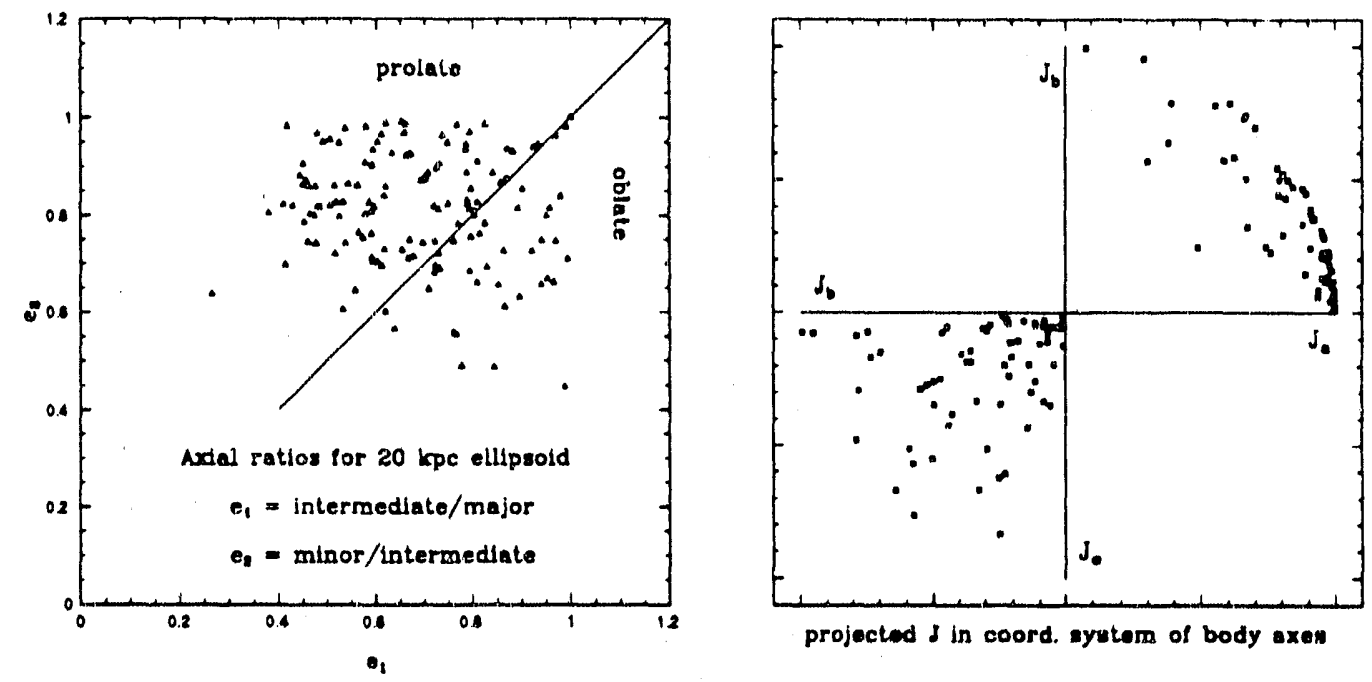

Figure 3. a.) The axial ratios of the 140 halos in the sample. Approximately $2 / 3$ of the halos lie on the prolate side of $e_{1}=e_{2}$.

b.) To demonstrate alignment of the angular momentum vector $\vec{J}$ with the minor axis $\vec{a}$ we have plotted $J_{a}=\hat{J} \cdot \hat{a}$ vs. $J_{b}=\hat{J} \cdot \hat{b}$ in the upper right corner $(\hat{J}=\vec{J} /|J|, \hat{a}=\vec{a} /|a|$, etc.). For a large fraction of the halos $\hat{J} \cdot \hat{a} \simeq 1$, that is, $\vec{J}$ and $\vec{a}$ are aligned. By contrast, in the plot of $J_{b}=\hat{J} \cdot \hat{b}$ vs. $J_{c}=\hat{J} \cdot \hat{c}$ in the lower left, most of the halos lie near the origin. That is, their angular momentum vector tends to be normal to the plane defined by the intermediate and major axes of the ellipsoid. Only halos with spin parameter $\lambda>0.04$ were used in this plot.

\section{(c.) Rotation and shape}

The alignment of the angular momentum in the halos is striking. The vectors are correlated among themselves in a given halo, and also tend to align with the minor axis of the halo. As shown in Fig. 3b, the vectors tend to point along the minor axis, and virtually none point along the major axis. The symbols in Fig. $3 \mathrm{~b}$ represent halos with a spin parameter larger than .04 . Approximately $75 \%$ of these halos have their total angular momentum vector pointing within $30^{\circ}$ of the minor axis. The effect is less pronounced for the the halos with $\lambda \leq 0.04$, but is still significant. Little correlation was seen between the degree of alignment and the eccentricity of the halo. 

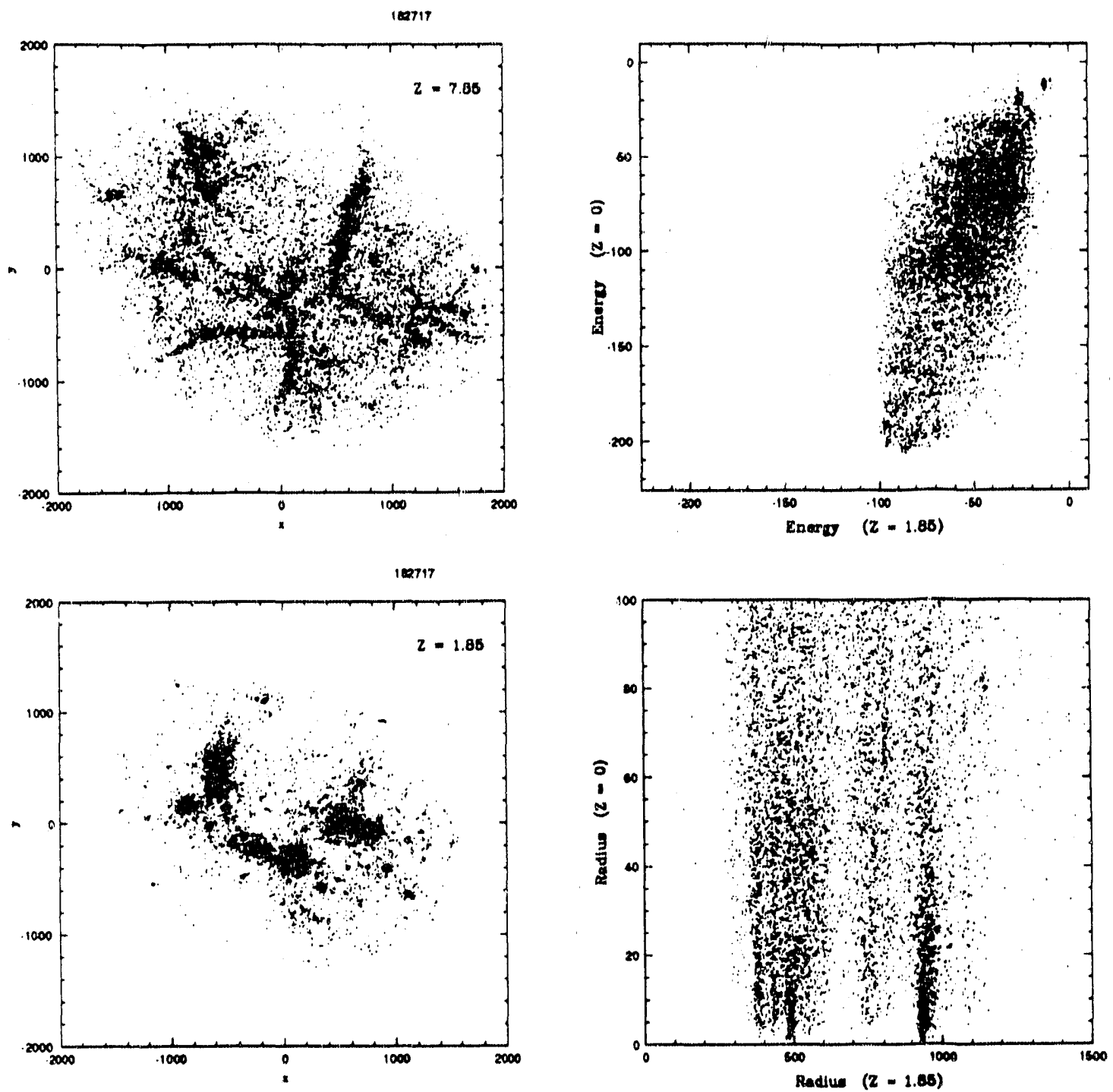

$1 \times 2717$

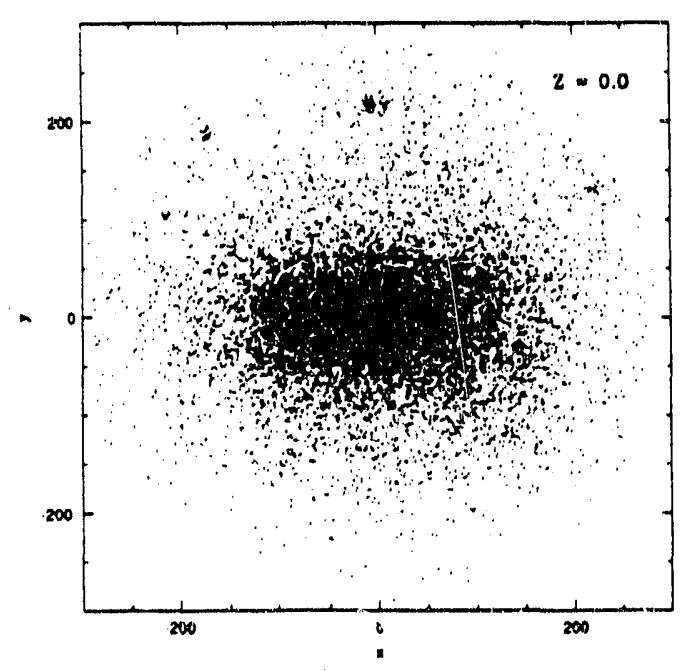

Figure 4. On the left are three snapshots, in comoving coordinates, of particles which end up in the selected final halo. On the upper right, a scatter plot of energy at $z=1.85$ vs. energy at $z=0$ suggests a correlation between the binding energies of particles at different epochs. The plot immediately above shows the distance from the center of mass at $z=1.85$ vs. the final radius. In disagreement with the assumptions of the secondary infall paradigm, no correlation is seen between the initial and final locations of particles. 


\section{DISCUSSION}

The evidence presented here supports the notion that halo formation is an organized process. The processes of tidal stripping and dynamical friction give rise to orderly deposition of mass, angular momentum and energy. The secondary infall paradigm $\mathrm{m}^{6,7,8}$ models collapse.as a self-similar process, in the course of which shells are deposited radially onto an already virialised core. Its predictions are in accord with the density profiles found in the numerical experiment. However, its primary assumption (layer by layer infall onto the central peak) is in violent disagreement with the formation process observed in the simulations. Fig. 4 supports the view that the collapse process appears to be primarily due to the merging of already collapsed objects. ${ }^{11,12}$ Quinn and Zurek ${ }^{13}$ have addressed this issue, and have $\mathrm{CO}$.jectured that the binding energy of the particles - and not their distance from the peak - is the important parameter. This also implies particles in the cores of progenicor clumps tend to end up in the core of the final halo." Regularities we have observed in the numerical experiments may lead to analytic models which relate density profiles, angular momenta and other parameters of the coilapsed halo to the initial cosmological or pre-merger coinditions.

We would like to thank James Kuyper for writing the assembly code for the force calculation subroutine and the Caltech Concurrent Supercomputer Facility for providing time on the MarkIII hypercube. One of us (MSW) was supported in part with a pre-doctoral fellowship by a grant from IGPP.

\section{References}

1. Ya. B. Zel'dovich, Astr. Ap., 5, 84-89, 1970.

2. G. Efstathiou, M. Davis, C. S. Frenk, and S. D. M. White, Ap. J. Suppl., 57, $241-260,1985$.

3. J. Barnes and P. Hut, Natuie, 324, 446-449, 1986.

4. F. R. Bouchet and L. Hernquist, Ap. J. Suppl., 68, 521-538, 1988.

5. J. K. Salmon, Parallel Hierarchical N-body Simulations, Ph. D. Thesis, CaliSornia Institute of Technology, 1991.

6. J. E. Gunn and J. R. Gott, Ap. J., 176, 1, 1972.

7. Y. Hoffman and J. Shaham, Ap. J., 297, 16-22, 1985.

8. J. A. Fillmore and P. Goldreich, Ap. J., 281, 1-8, 1984.

9. P. J. Quinn, J. K. Salmon, and W. H. Zurek, Nature, 322, 329-335, 1986.

10. G. Efstathiou, C. S. Frenk, S. D. M. White, and M. Davis, M.N.R.A.S, 235, 715-748, 1988 .

11. W. H. Zurek, F. J. Quinn, and J. K. Salmon, Ap. J., 330, 519-534, 1988.

12. P. J. Quinn, W. H. Zurek, J. K. Salmon, and M. S. Warren, "The Formation of Halos via Mergers: the organized and organizing dynamics of mergers", in Proceedings of 1989 Heidelberg Conference on Dynamics and Interactions of Galaxies, ed. A. Toomre and R. Wielen, Springer-Verlag, 1990.

13. P. J. Quinn and W. H. Zurek, Ap. J., 331, 1-18, 1988. 

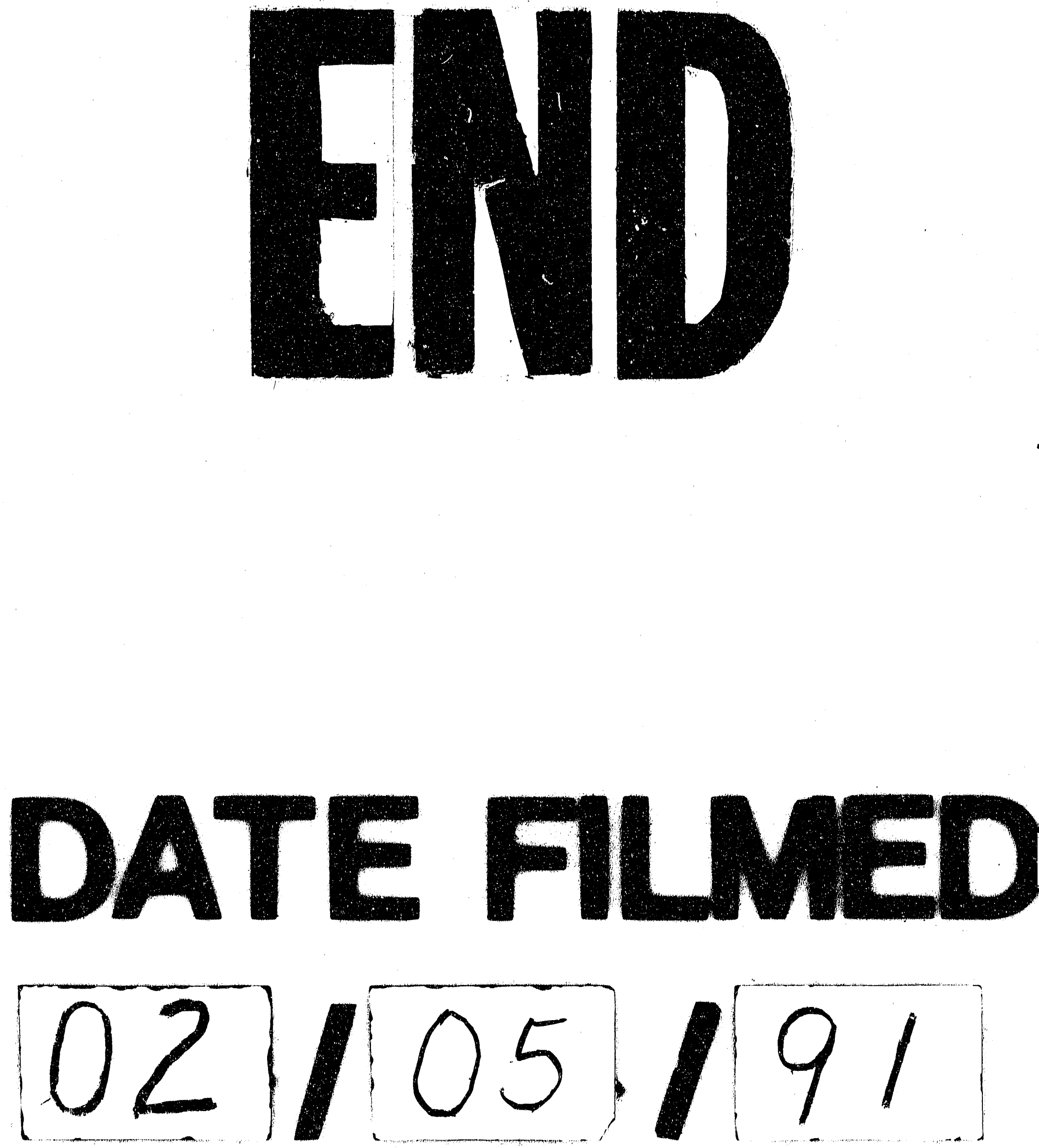
